ISSN 0103-5150

Fisioter. Mov., Curitiba, v. 25, n. 2, p. 291-299, abr./jun. 2012

Licenciado sob uma Licença Creative Commons

\title{
Cadeia cinética aberta versus cadeia cinética fechada na reabilitação avançada do manguito rotador
}

\author{
Open kinetic chain versus closed kinetic chain \\ in advanced rehabilitation rotator cuff
}

\section{Rudiel Luciano Boeck ${ }^{[a]}$, Marcelo Baptista Döhnert ${ }^{[b]}$, Tiago Sebastiá Pavão ${ }^{[c]}$}

[a] Acadêmico de Graduação do curso de Fisioterapia da Universidade Luterana do Brasil (ULBRA), Torres, RS - Brasil, e-mail: rudiel3b@hotmail.com

[b] Professor do curso de Graduação em Fisioterapia da Universidade Luterana do Brasil (ULBRA), Torres, RS - Brasil, e-mail: mdohnert@ig.com.br

[c] Professor do curso de Graduação em Fisioterapia da Universidade Luterana do Brasil (ULBRA), Torres, RS - Brasil, e-mail: tiagospavao@hotmail.com

\section{Resumo}

Introdução: Síndrome do impacto do ombro é uma alteração osteomuscular prevalente que leva a uma redução significativa da saúde e à incapacidade funcional. Esta lesão é causada pelo uso repetitivo dos braços acima da linha do ombro ou condição patológica em que ocorre irritação do tendão supraespinhoso secundária a uma abrasão em sua superfície pelo terço anterior do acrômio. Fisioterapia é, muitas vezes, a primeira opção de tratamento, embora sua eficácia ainda esteja em debate. Clinicamente, a reabilitação em cadeia cinética tem sido eficaz na restauração da função do ombro com bons resultados terapêuticos. Objetivos: Avaliar a efetividade de um protocolo de reabilitação do ombro em cadeia cinética fechada para síndrome de impacto do manguito rotador. Materiais e métodos: Ensaio clínico randomizado de equivalência com 20 pacientes que apresentam lesão grau I e II de manguito rotador na classificação de Neer. Pacientes foram divididos em dois grupos: grupo I, com protocolo de exercícios em Cadeia Cinética Fechada (CCF), e grupo II, com exercícios em Cadeia Cinética Aberta (CCA). Os pacientes foram submetidos a 20 sessões, três vezes por semana, e foram avaliados quanto à dor, mobilidade ativa, passiva, força muscular, atividade elétrica muscular e funcionalidade. Essa avaliação ocorreu em três momentos: inicialmente, com 10 sessões e ao fim do tratamento. Para a análise estatística, foram utilizadas medidas paramétricas, como o teste t-Student (comparação entre os grupos) e ANOVA para medidas repetidas 
(comparação dentro de cada grupo), e medidas não paramétricas (Kruskal Wallis e teste de Friedman). Resultados: Ambos os grupos apresentaram resultados significativos quanto aos escores obtidos na escala UCLA e Constant. A mobilidade ativa do ombro lesado aumentou no grupo CCF nos movimentos de flexão $(p=0,01)$, rotação externa $(p=0,000)$ e rotação interna $(p=0,000)$. 0 movimento de abdução melhorou nos dois grupos ( $p=0,02$ no grupo CCF e 0,04 no grupo CCA). A força muscular de flexão e abdução mostrou um aumento apenas no grupo CCF, enquanto que nos movimentos de rotação externa e interna esses aspectos foram significativos em ambos os grupos. Conclusão: Constatou-se que exercícios em CCF apresentam melhoras importantes na mobilidade, funcionalidade e força para os pacientes com síndrome de impacto de ombro.

Palavras-chave: Síndrome de colisão do ombro. Terapia por exercício. Fisioterapia.

\section{Abstract}

Introduction: Shoulder impingement syndrome is a prevalent musculoskeletal alteration that leads to a significant reduction of health and to functional disability. This injury is caused by repetitive use of arms above the shoulder line or pathological condition to which the supraspinatus tendon irritation occurs secondary to an abrasion on its surface by the anterior third of the acromion. Physiotherapy is often the first choice of treatment, although its effectiveness is still under debate. Clinically, the kinetic chain rehabilitation has been effective in restoring shoulder function with good therapeutic results. Objectives: To evaluate the effectiveness of a protocol for rehabilitation of the shoulder in a closed kinetic chain for impact syndrome rotator cuff. Materials and methods: Randomized clinical trial of equivalence with 20 patients with grade I and II lesions of the rotator cuff in the classification of Neer. Patients were divided into two groups: group I, with the exercise protocol in closed kinetic chain (CKC), and group II, with exercises in open kinetic chain (OKC). Patients underwent 20 sessions, three times a week, and were evaluated on pain, mobility, active, passive, muscular strength, muscular activity and functionality. This evaluation occurred three times: initially, with 10 sessions and at the end of treatment. Statistical analysis used parametric measures such as the t-Student test (comparison between groups) and ANOVA for repeated measures (comparison within each group), and nonparametric measures (Kruskal Wallis and Friedman test). Results: Both groups showed significant results regarding the scores obtained in the UCLA and Constant scale. The active mobility of the shoulder increased in the injured group CCF in flexion ( $p=0.01)$, external rotation $(p=0.000)$ and internal rotation $(p=0.000)$. The movement of abduction improved in both groups ( $p=0.02$ in CCF group and 0.04 in CCA group). Muscle strength of flexion and abduction showed an increase only in the CCF group, while in the rotation movements were external and internal significant in both groups. Conclusion: It was concluded that CKC exercises have major improvements in mobility, functionality and strength for patients with shoulder impact syndrome.

Keywords: Shoulder impingement syndrome. Exercise therapy. Physical therapy.

\section{Introdução}

Dentre as lesões do ombro que provocam dor e impotência funcional, a síndrome de impacto do manguito rotador se destaca e foi descrita por Charles Neer pela primeira vez em 1972 (1). Caracteriza-se pelo uso repetitivo dos braços acima da linha do ombro, condição patológica em que ocorre a irritação do tendão supraespinhoso secundária a uma abrasão em sua superfície pelo terço anterior do acrômio $(2,3)$. Neer também relata a importância da superfície interna do tendão e as potenciais mudanças que podem ocorrer pela fricção repetitiva e irritação deste no estágio três na classificação do processo de impacto (4). A altura do espaço subacromial em ombros saudáveis está entre 9 e 10 milímetros, sendo que medidas radiográficas menores que 6 milímetros são consideradas patológicas para compressão do manguito rotador. A espessura normal do tendão do manguito rotador nesta área está entre cinco e seis milímetros (5). 
0 protocolo de cadeia cinética fechada é um modelo biomecânico utilizado para analisar muitas atividades esportivas e gestos biomecânicos, representando o corpo como um sistema coerente de segmentos interdependentes, muitas vezes trabalhando em uma sequência de proximal para distal, para dar uma ação desejada no segmento distal. Isso reforça os padrões de movimento normal e reduz o desafio de aprender novos movimentos durante a reabilitação, numa sequência coerente com a função biomecânica da extremidade superior (6-9). Giordano et al. compartilham da opinião de Greve (1995 a, b) e de outros autores (Neer II, 1995; Morelli \& Vulcano, 1993) de que o tratamento a SIO deve ser inicialmente clínico, independentemente da presença de alterações anatômicas envolvidas na gênese da doença (10). 0 objetivo do estudo foi avaliar a efetividade de um protocolo de reabilitação do ombro em cadeia cinética fechada para síndrome de impacto do manguito rotador.

\section{Materiais e métodos}

\section{Delineamento}

Ensaio clínico randomizado de equivalência realizado com pacientes com diagnóstico de síndrome de impacto de ombro grau I e II, entre os meses de agosto e novembro de 2010.

Ética

O estudo foi aprovado pelo Comitê de Ética e Pesquisa em Seres Humanos e Animais da Universidade Luterana do Brasil, sob o registro 2010-213H. Explicou-se a todos os participantes o objetivo da pesquisa, os critérios de inclusão e de exclusão, os procedimentos experimentais e de avaliação. Todos os participantes assinaram o termo de consentimento livre e esclarecido.

\section{Critérios de elegibilidade}

A população abordada foi composta por pessoas acometidas por síndrome de impacto de manguito rotador em grau I e II inicial na classificação de Neer e com solicitação de tratamento fisioterapêutico.
Foram incluídos no estudo pacientes com diagnóstico clínico de síndrome de impacto concedido por médico traumatologista, com a presença de, pelo menos, três testes clínicos positivos para síndrome de impacto e manguito rotador; presença de ressonância magnética apresentando quadro inflamatório, espessamento de manguito rotador e cabo longo do bíceps; ou ecografia apresentando quadro inflamatório, espessamento de manguito rotador e cabo longo do bíceps; não estar realizando previamente algum outro tipo de modalidade fisioterapêutica; e ter assinado o Termo de Consentimento Livre e Esclarecido. Foram excluídos do estudo sujeitos com presença de cirurgia ou outro tipo de lesão prévia no ombro afetado, diagnóstico de síndrome de impacto grau III de Neer, indicação cirúrgica concedida pelo médico traumatologista e ter três faltas consecutivas não justificadas ao protocolo experimental.

\section{Randomização}

Os pacientes foram divididos aleatoriamente em dois grupos:

Grupo I - recebendo intervenção por meio de exercícios em CCF + medicamento(s) prescrito(s) pelo médico traumatologista + orientações domiciliares + crioterapia domiciliar;

Grupo II - recebendo intervenção por meio de exercícios em CCA + medicamento(s) prescrito(s) pelo médico traumatologista + orientações domiciliares + crioterapia domiciliar.

\section{Protocolo de avaliação}

\section{Testes clínicos}

Os testes clínicos, além de terem sido utilizados como critério diagnóstico, serviram como parâmetro para análise da evolução terapêutica de cada grupo. Foram utilizados os testes de Neer, Jobe, HawkinsKennedy e Yocum.

\section{Amplitude de movimento}

A avaliação da amplitude de movimento foi realizada com a utilização de um goniômetro como 
orientações do manual de goniometria (11), no qual tínhamos um braço fixo e o móvel que acompanhava o sentido do arco de movimento até o máximo de amplitude que o paciente conseguia.

\section{Qualidade de vida}

A qualidade funcional e o grau de comprometimento foram avaliados por meio dos questionários validados de UCLA, o qual avalia dor, função, mobilidade de flexão ativa, força muscular (teste manual) e satisfação do paciente, e a escala de CONSTANT, que avalia dor, atividades de vida diária (AVDs) (limitações no trabalho, lazer, dormir, função do braço) e média de movimentos (flexão, abdução, rotação externa - RE, rotação interna - RI).

\section{Atividade eletromiográfica dos músculos} do manguito rotador

Outro instrumento de avaliação utilizado foi a eletromiografia de superfície (EMG). Ela determina o início de ativação muscular e avalia a coordenação ou o desequilíbrio dos diferentes músculos envolvidos $(12,13)$. Utilizou-se um eletromiógrafo de dois canais modelo EMG Retrainer, marca Chattanooga Group ${ }^{\circledR}$ com eletrodos de superfície autoadesivos e descartáveis. A coleta dos sinais foi iniciada com a tricotomia dos pelos quando necessário, limpeza da pele com algodão embebido em álcool $70 \%$, seguida da colocação dos eletrodos, guiado pela disposição das fibras musculares e prova de função dos músculos analisados. 0 sinal foi captado em contração isotônica isométrica voluntária máxima e expresso em microvolts $(\mu V)$ (12). Foram avaliadas a atividade elétrica dos músculos supraespinhoso, infraespinhoso, deltoide anterior, deltoide médio, deltoide posterior e bíceps braquial. A coleta dos dados ocorreu simultaneamente à análise da força muscular.

\section{Força muscular}

A contração isométrica voluntária máxima (CIVM) foi avaliada simultaneamente à realização da eletromiografia de superfície. Foram observados os movimentos de rotação externa, rotação interna, flexão, abdução e extensão. Foi utilizado um dinamômetro modelo push-pull, marca Chattanooga Group ${ }^{\circledR} .0$ aparelho foi estabilizado pelo avaliador, não permitindo que o paciente executasse o movimento durante o teste de contração.

\section{Protocolo de intervenção}

Os pacientes receberam o protocolo de exercícios três vezes por semana, divididos em subgrupos de quatro pacientes para dinamizar o atendimento. 0 protocolo teve duração média de 50 minutos para cada subgrupo e contou com mais 12 minutos de crioterapia ao final do protocolo.

\section{Intervenção CCA}

- Exercícios pendulares de Codman;

- Flexão: em decúbito dorsal, elevando o braço até o limite da dor, repetir o gesto 20 vezes;

- Rotação interna: em decúbito dorsal, trazer o braço de rotação neutra para interna, repetir o gesto 20 vezes;

- Rotação externa: em decúbito dorsal, trazer o braço de rotação neutra para externa, repetir o gesto 20 vezes;

- Abdu-adução: em decúbito dorsal, trazer o braço para junto do corpo, repetir o gesto 20 vezes;

- Abdu-adução horizontal: em decúbito dorsal, com os braços abertos, bater palma na altura do peito, repetir o gesto 20 vezes;

- Elevação em polia, sentado, 60 repetições;

- Flexão: sentado, elevando o braço até o limite da dor, repetir o gesto 20 vezes;

- Rotação interna: em ortostase, trazer o braço de rotação neutra para interno ("coçar as costas"), até o limite da dor, repetir o gesto 20 vezes;

- Rotação externa: sentado trazer o braço de rotação neutra para externa ("pedir carona"), até o limite da dor, repetir o gesto 20 vezes;

- Abdu-adução: sentado, "bater palmas", até o limite da dor, repetir o gesto 20 vezes;

- Abdu-adução horizontal: sentado, com os braços abertos, "bater palmas" na altura do peito, repetir o gesto 20 vezes;

- Crioterapia após exercício durante 12 minutos. 
Intervenção CCF

- Flexão: de pé, mãos apoiadas na parede, realizando movimento de reverência ("agachamento"), até o limite da dor, repetir o gesto 20 vezes;

- Extensão: em pé, de costas para a parede, mãos apoiadas na mesma, afastando as costas da parede, até o limite da dor, repetir o gesto 20 vezes;

- Rotação interna: em pé, mão apoiada na parede com ombro em extensão (de costas para a parede), paciente realiza a inclinação em diagonal para trás e para o lado da mão apoiada na parede, até o limite da dor, repetir o gesto 20 vezes;

- Rotação externa: de pé, mãos apoiada no marco de uma porta ou pilar, cotovelo e ombro flexionados a 90 graus, realizar uma inclinação do tronco à frente apoiando-se sobre o membro em exercício, até o limite da dor, repetir o gesto 20 vezes;

- Abdu-adução: em ortostase, apoiar as mãos nas barras paralelas e agachar, até o limite da dor, repetir o gesto 20 vezes;

- Abdu-adução horizontal: de quatro apoios (sobre a bola suíça, para iniciar), realizando inclinações laterais, até o limite da dor, repetir o gesto 20 vezes;

- Flexão: de quatro apoios (sobre a bola suíça, para iniciar), realizando movimento de reverência, até o limite da dor, repetir o gesto 20 vezes;

- Extensão: decúbito dorsal, apoiado nos cotovelos erguendo as costas do tatame, até o limite da dor, repetir o gesto 20 vezes;

- Crioterapia após exercícios durante 12 minutos.

\section{Análise estatística}

Para a análise estatística, utilizou-se o SPSS (Statistical Package for the Social Sciences) versão 17.0. Os dados foram expressos em frequência, média e desvio padrão. Para avaliar a homogeneidade dos grupos, foi utilizado o teste de qui quadrado. Já para a análise dos resultados de intervenção foi utilizado o teste t-Student para as medidas paramétricas entre os grupos de intervenção e a análise de variância (ANOVA) one-way para medidas repetidas, seguido pelo teste post hoc de Bonferroni para comparação das médias pré, parcial e pós-intervenção dentro de cada grupo. Para as medidas não paramétricas, foram utilizados os testes de Kruskal Wallis e Friedman. 0 nível de significância estabelecido para o teste estatístico é de $\mathrm{p}<0,05$.

\section{Resultados}

Entre agosto e novembro de 2010, 14 pacientes foram incluídos no estudo. A média de idade total foi de $55,57 \pm 11,85$ anos, sendo que no grupo CCF a média de idade foi de $54,71 \pm 12,84$ anos, e de 56,43 $\pm 11,74$ anos para o grupo CCA. No grupo CCF havia sete mulheres e, no grupo CCA, seis eram do sexo feminino e um do masculino. Não se observou diferenças significativas entre ambos os grupos nas suas características ( $p>0,05)$.

Para avaliar a função foram utilizadas duas escalas funcionais validadas: escala de UCLA (Universidade da Califórnia, Los Angeles) e Constant. Houve melhora no escore total da escala UCLA em ambos os grupos após a intervenção. No grupo CCF, o escore inicial era $12,42 \pm 1,49$ e, ao fim do protocolo, foi de $27 \pm 2,3$ ( $\mathrm{p}=0,003$ ). Já no grupo CCA, o escore inicial foi de $18 \pm 3,4$ e, ao fim da intervenção, foi de $32,66 \pm 0,42$ $(p=0,02)$ (Figura 1). Na escala de Constant também se verificou melhora dos escores de ambos os grupos, porém no grupo CCF esta melhora já ocorreu a partir da décima sessão. Os sujeitos do grupo CCF apresentaram, inicialmente, um escore $31,71 \pm 3,68$ e, ao final da intervenção, $62,42 \pm 4,49(\mathrm{p}=0,002)$. Já no grupo CCA, o escore inicialmente foi de 36,83 $\pm 20,91$ e, ao final da intervenção, foi de 74,67 $\pm 2,65$ ( $p=0,017$ ) (Figura 2).

A amplitude de movimento (ADM) ativa do ombro lesado, realizada com um goniômetro universal, mostrou um aumento significativo no grupo CCF para os movimentos de flexão $(p=0,011)$, abdução $(p=0,021)$, rotação externa $(p=0,000)$ e rotação interna $(p=0,000)$. Já no grupo CCA, apenas a abdução apresentou melhora $(p=0,04)$. Todos os planos de movimentos, no grupo CCF, obtiveram melhora significativa já nas 10 primeiras sessões. A avaliação da ADM passiva mostrou resultados semelhantes à ADM ativa. Apenas o grupo CCF obteve resultados expressivos de melhora na abdução $(p=0,025)$, rotação externa $(p=0,013)$ e interna $(p=0,043)$. Na flexão, não se obteve significância, pois os pacientes já apresentavam na avaliação inicial uma ADM de 127,85 ${ }^{\circ}$, não gerando significância no aumento da amplitude. 


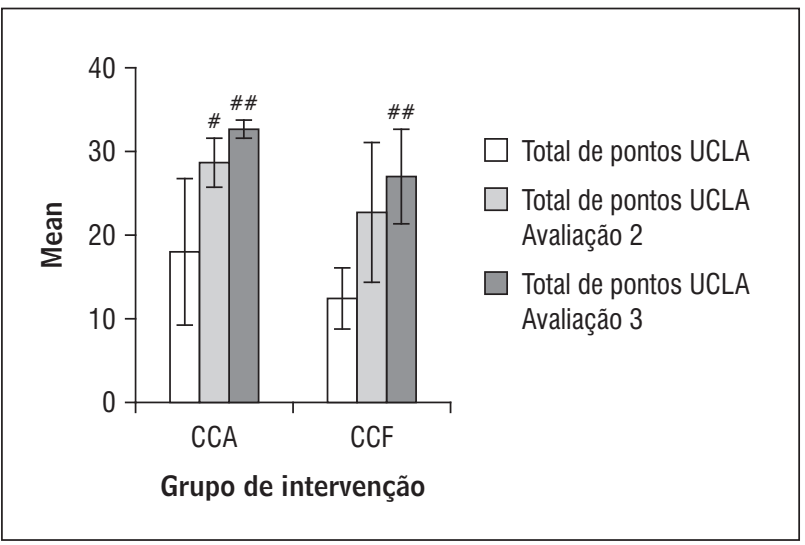

Figura 1 - Análise da avaliação UCLA ambos os grupos Legenda: \# $p=0,02$ avaliação inicial para avaliação parcial com dez sessões; \#\# $p=0,003$ para grupo CCF e $p=0,022$ para grupo CCA avaliação inicial para avaliação final.

A força muscular, avaliada por meio da dinamometria, melhorou em todos os planos de movimento no grupo CCF. A força muscular inicial para flexão foi de 2,91 kg e aumentou para 7,75 kg ao fim do protocolo $(p=0,024)$. A força muscular de abdução aumentou de 3,16 kg para 7,41 kg $(p=0,05)$ (Figura 3). Na rotação externa, a força muscular passou de $2,25 \mathrm{~kg}$ para $8,08 \mathrm{~kg}$ ao final do protocolo $(\mathrm{p}=$ 0,001 ) (Figura 4).

Por fim, a força de rotação interna passou de 2,83 $\operatorname{kg}$ para $8,33 \mathrm{~kg}(\mathrm{p}=0.001)$ (Figura 5). No grupo CCA, apenas a força dos movimentos rotacionais apresentou melhora ao final do protocolo. A rotação externa inicial era de $3,80 \mathrm{~kg}$ e progrediu para $7,9 \mathrm{~kg}$ ao final do protocolo $(\mathrm{p}=0.028)$ (Figura 4). Já a rotação interna apresentava $5,2 \mathrm{~kg}$ e melhorou para $8,7 \mathrm{~kg}$ ( $p=0.009$ ) (Figura 5).

A ativação de unidades motoras, avaliada por meio da eletromiografia de superfície, não mostrou resultados estatísticos significativos. Observou-se, porém, médias de sinais elétricos que acompanharam fidedignamente o ganho de força muscular de ambos os grupos.

Por meio dos testes clínicos específicos, conseguimos analisar o grau de acometimento e, com uma medida qualitativa, observar a evolução final dos pacientes em relação à primeira avaliação.

0 teste Neer era inicialmente positivo em 11 sujeitos do estudo (todos os sete no grupo CCF e quatro no grupo CCA). Ao final do tratamento, quatro ainda eram positivos no CCF e apenas um no CCA.

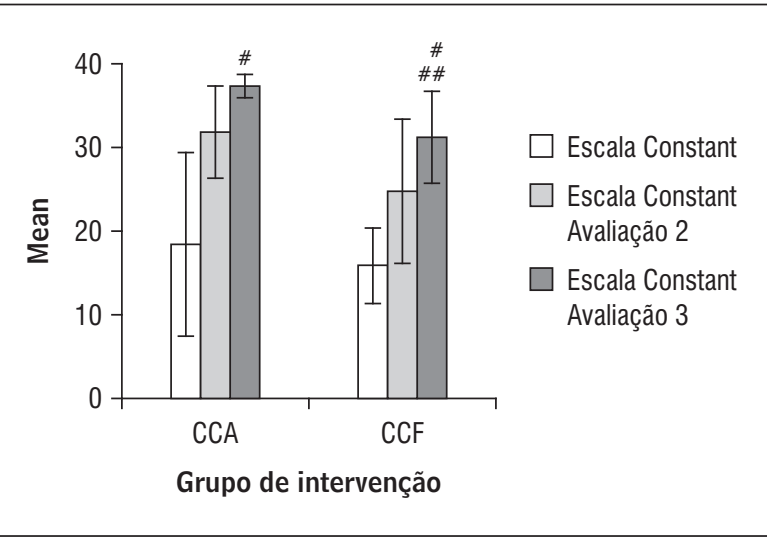

Figura 2 - Análise dos pontos da escala de Constant ambos os grupos

Legenda: \# $p=0,002$ para grupo CCF e $p=0,017$ para grupo CCA avaliação inicial para avaliação final; \#\# $p=0,026$ avaliação parcial com dez sessões para avaliação final.

No teste de Hawkins, todos os sete sujeitos do grupo CCF eram positivos, sendo que, ao final da intervenção, todos os casos se tornaram negativos. Já no grupo CCA, três eram positivos e, após a intervenção, um caso ainda era positivo.

0 teste de Yocum mostrava positividade em seis casos positivos no grupo CCA e todos os sete no CCF. Ao final da intervenção, apenas um positivo no CCA e quatro ainda eram positivos no CCF.

Finalmente, no teste de Jobe, todos os 14 sujeitos do estudo apresentavam teste positivo no início do estudo. Na reavaliação final, cinco em cada grupo tornaram-se negativos.

\section{Discussão}

Vários autores têm pesquisado a reabilitação de pacientes com SIO através de programa de reabilitação através de exercícios em CCF. Os protocolos conservadores deste tipo de pacientes normalmente abordam protocolos que utilizam exercícios em CCA.

Neste estudo, avaliamos a recuperação funcional através das escala UCLA e Constant. Çitaker et al. (14) avaliaram a funcionalidade de indivíduos com síndrome do impacto, utilizando a UCLA, e encontraram melhoras significantes após 20 sessões de intervenção. Ambos os grupos do estudo apresentaram melhora significativa dos escores funcionais em ambas as escalas. Vários estudos corroboram com os nossos achados. Glasoe et al. (15) investigaram a eficácia de 


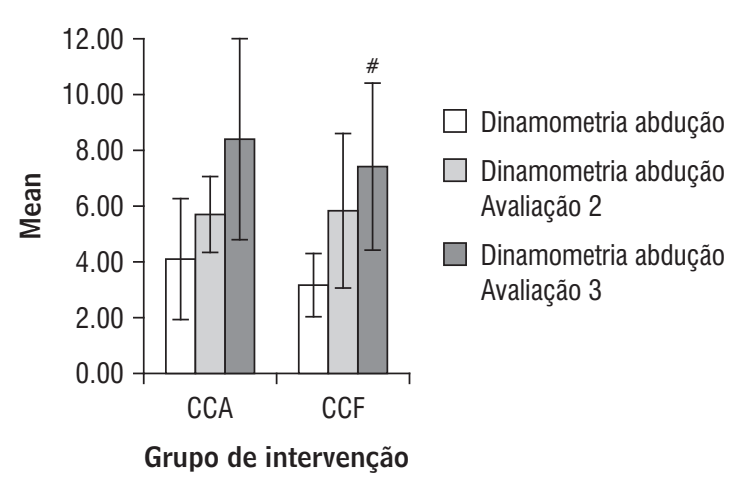

Figura 3 - Análise da força dinamométrica de abdução do ombro (kg)

Legenda: \# $\mathrm{p}=0,05$ avaliação inicial para avaliação final.

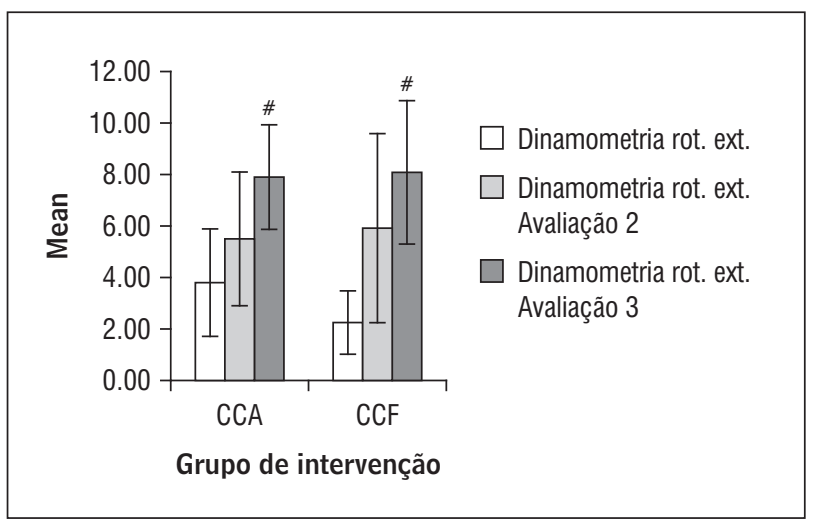

Figura 4 - Análise da força dinamométrica de rotação externa do ombro $(\mathrm{kg})$

Legenda: \# $p=0,001$ grupo CCF e $p=0,028$ grupo CCA avaliação inicial para avaliação final.

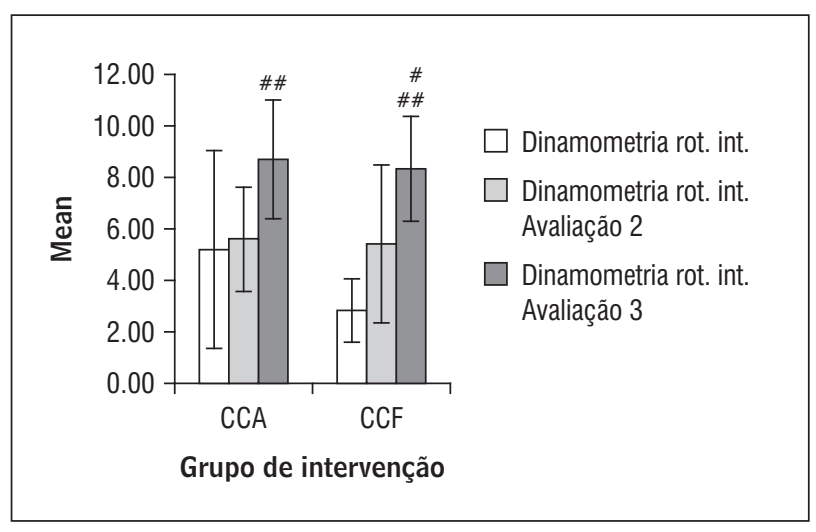

Figura 5 - Análise da força dinamométrica de rotação interna do ombro $(\mathrm{kg})$

Legenda: \# $p=0,001$ avaliação inicial para avaliação final; \#\# $p=0,04$ grupo CCF e $p=0,009$ grupo CCA avaliação parcial com dez sessões para avaliação final. um protocolo de tratamento pra SIO com CCA, por meio da escala UCLA, e constataram melhora siginificativa após a intervenção. Lech et al. (16) analisaram o sucesso de uma abordagem fisioterapêutica conservadora para pacientes com SIO e obtiveram $69,2 \%$ de bons e excelentes resultados na escala UCLA. Barbosa, Goes e Fonseca (17) abordaram pacientes com SIO por meio de exercícios em CCA, verificando uma melhora do escore UCLA: de 14,57 na avaliação inicial para 28,0 na avaliação final. Em nosso estudo, os escores iniciais foram de 12,42 (CCF) e 18,00 (CCA), progredindo para $27,00(\mathrm{CCF})(\mathrm{p}=0,003)$ e 32,67 ( $\mathrm{p}=0,022)$.

Levando em consideração a escala de Constant, outro estudo avaliou a influência da mobilização articular em pacientes com tendinopatia de bíceps e supraespinhoso e encontrou escores significativos na escala de Constant (17). San Segundo et al. (18) utilizaram a escala de Constant para avaliar a melhora de pacientes com SIO submetidos a um protocolo utilizando a terapêutica do ultrassom e verificaram que o escore inicial era de 49,4 e evoluiu para 61,3 ( $p=0,001)$. Em nosso estudo, os escores iniciais foram de 31,71 (CCF) e 36,83 (CCA), progredindo para $62,43$ (CCF) (p = 0,002) e 74,66 (CCA) ( $p=0,017)$.

Tanto a ADM ativa quanto a passiva obtiveram ganho expressivo no grupo tratado com exercícios em CCF, enquanto que no grupo CCA apenas a abdução melhorou. Uma revisão Cochrane concluiu que a terapia por exercícios por meio de CCA para o manguito rotador é benéfica, tanto a curto como a longo prazo, no que diz respeito a uma maior valorização da função e à progressão de abdução (19). Outro estudo refere que o efeito tem sido visto até 2,5 anos após a conclusão do tratamento. No entanto, há evidências limitadas para sugerir qual é o regime de exercício ótimo, ou a frequência ou a intensidade do programa para aprimorar a terapêutica (20).

Neste estudo, verificamos uma melhora expressiva da força muscular em todos os planos de movimento para o grupo CCF, enquanto o grupo CCA apresentou apenas melhora da força nos movimentos de rotação externa e interna. Glasoe et al. (15) avaliaram o ganho de força por meio de dinamometria isocinética pré e pós-intervenção, e constataram um ganho de $93 \%$ na força do membro contralateral ao final do estudo. Araújo et al. (21) avaliaram a atividade eletromiográfica dos músculos do ombro e cintura escapular durante um programa de exercícios em CCF em 20 sujeitos, detectando um excelente índice de confiabilidade intragrupo com essa técnica de exame. 
Em nosso estudo, apesar de não termos observado um resultado significativo nas médias da atividade eletromiográfica, notamos que o comportamento da atividade elétrica de cada grupo muscular avaliado acompanhou os resultados apresentados na avaliação da contração isométrica voluntária máxima (CIVM).

\section{Conclusão}

Ambos os protocolos de intervenção apresentaram resultados satisfatórios na reabilitação da SIO. Verificou-se que o grupo de intevenção tratado com um programa de exercícios em CCF apresentou resultados mais expressivos quanto ao ganho de mobilidade articular, funcionalidade, força e ativação muscular do manguito rotador, em comparação ao programa de exercícios em CCA. Acreditamos que o tamanho pequeno da amostra e a falta de um acompanhamento destes pacientes por um período maior tenha sido um fator limitante do estudo. Sugerimos a realização de novos estudos com amostragens maiores para a confirmação de tais resultados.

\section{Lista de abreviações}

SIO: síndrome de impacto do ombro;

CCA: cadeia cinética aberta;

CCF: cadeia cinética fechada;

ADM: amplitude de movimento;

UCLA: Universidade da Califórnia, Los Angeles;

$\mu \mathrm{V}$ : microvolts;

RE: rotação externa;

RI: rotação interna;

AVDs: atividades de vida diária;

CIVM: contração isométrica voluntária máxima.

\section{Conflitos de interesse}

Os autores declaram não haver conflitos de interesse entre as partes.

\section{Referências}

1. Neer CS. Anterior acromioplasty for the chronic impingement syndrome in the shoulder: a preliminary report. J Bone Joint Surg Am. 1972;54(1):41-50.
2. Morison DS, Greenbaum BS, Einhorn A. Shoulder impingement. Orthop Clin North Am. 2000;31(2):285-93.

3. Lewis JS, Green AS, Dekel S. The aetiology of subacromial impingement syndrome. Physiotherapy. 2001; 87(9):458-69.

4. Neer CS. Impingement lesions. Clin Orthop Relat Res. 1983;(173):70-7.

5. Donatelli RA. Impingement syndrome and impingement related instability. Physical therapy of the shoulder. New York: Churchill Livingstone; 1997. p. 229-56.

6. McMullen J, Timothy L. A kinetic chain approach for shoulder rehabilitation. J Athl Train. 2000;35(3):329-37.

7. Feltner ME, Dapena J. Three-dimensional interactions in a two-segment kinetic chain, part I: general model. Int J Sport Biomech. 1989;5:403-19.

8. Cordo PJ, Nashner LM. Properties of postural adjustments associated with rapid arm movements. J Neurophysiol. 1982;47:287-308.

9. Zattara M, Bouisset S. Posturo-kinetic organisation during the early phase of voluntary upper limb movement, 1: normal subjects. J Neurol Neurosurg Psychiatry. 1988;51(7):956-65.

10. Giordano M, Giordano V, Giordano BLH, Giordano N. J. Tratamento conservador da síndrome do impacto subacromial. Acta Fisiátrica. 2007;7:13-9.

11. Marques AP. Manual de goniometria. 2a ed. São Paulo: Manole; 2003.

12. Lehman GJ, MacMillan B, MacIntyre I, Chivers M, Fluter M. Shoulder muscle EMG activity during push up variations on and off a Swiss Ball. Dyn Med. 2006;5:7.

13. Ling SM, Conwit RA, Talbot L, Shermack M, Wood JE, Dredge EM, et al. Electromyographic patterns suggest changes in motor unit physiology associated with early osteoarthritis of the knee. Osteoarthritis Cartilage. 2007;15(10):1134-40.

14. Çitaker S, Takiran H, Akdur H, Önel Ü, Ekici G. Comparison of the mobilization and proprioceptive neuromuscular facilitation methods in the treatment of shoulder impingement syndrome. The PainClinic. 2005;17(2):197-202.

15. Glasoe WM, Fisher CJ, Murthy D. Treatment protocol for an acute large rotator cuff repair. Physiotherapy. 2004;90(4):217-20. 
16. Lech O, Neto CV, Severo A. Tratamento conservador das lesões parciais e completas do manguito Rotador. Acta Ortop Brás. 2000;8(3):144-56.

17. Barbosa RI, Goes R, Mazzer N, Fonseca MCR. A influência da mobilização articular nas tendinopatias dos músculos bíceps braquial e supra-espinal. Rev Bras Fisioter. 2008;12(4):298-303.

18. San Segundo RM, Molins J, Valdés M, Fernández TR. Tratamiento conservador del síndrome subacromial. Ultrasonidos frente a placebo. Um ensayo clínico. Rehabilitación. 2008;42(2):61-6.

19. Michener LA, Walsworth MK, Burnet EN. Effectiveness of rehabilitation for patients with subacromial impingement syndrome: a systematic review. J Hand Ther. 2004;17(2):152-64.
20. van der Windt DA, van der Heijden GJ, van den Berg SG, ter Riet G, de Winter AF, Bouter LM. Ultrasound therapy for musculoskeletal disorders: a systematic review. Pain. 1999;81(3):257-71.

21. Araújo RC, Tucci HT, Andrade R, Martins J, BevilaquaGrossi D, Oliveira AS. Reliability of electromyographic amplitude values of the upper limb muscles during closed kinetic chain exercises with stable and unstable surfaces. J Electromyogr Kinesiol. 2009;19(4):685-94.

Recebido: 03/04/2011

Received: 04/03/2011

Aprovado: 02/08/2011

Approved: 08/02/2011 